\title{
Simple Camera Calibration for Light Measurements
}

\author{
João C. Aparício Fernandes ${ }^{(1)}$, José A. B. Campos Neves, Manuel J. Sepúlveda Freitas ${ }^{(2)}$, João Luís Afonso ${ }^{(3)}$ \\ ${ }^{(1,2,3)}$ Dept. Industrial Electronics, Universidade do Minho, Campus de Azurém, Guimarães, Portugal \\ aparicio@dei.uminho.pt, jacn@netcabo.pt,.mjsf@dei.uminho.pt, jla@dei.uminho.pt
}

\begin{abstract}
The availability and decreasing prices of digital photographic cameras using megapixel sensors could point to its use as high quality light measuring devices if simple and effective calibration procedures could be put to work.

This paper proposes such a system, using a common digital camera combined with low cost reference color charts. The procedure to obtain the response function of the camera, the production of the color chart and its calibration is presented.

In this way, it is possible to obtain light measurements otherwise only possible with sophisticated and expensive equipments.
\end{abstract}

\section{INTRODUCTION}

Digital photographic cameras are widely available today at low prices due to their large scale production. Improved systems are constantly introduced to the market at lower prices than its predecessors. The light sensors these cameras use are sophisticated light measuring devices, unavailable a few years ago. However, these systems are aimed for photographic purposes and their technical characteristics are deeply buried in the assembled camera. The intended results are pictures and the light information data is processed according to specifications and methods of the equipment manufacturer that constitute his trade secrets.

Also the scale used for digital images is a non-linear relationship - the gamma-law- that further complicates the process, mainly because it is often disregarded [1].

On the other hand, light measurements must deal with the distribution of the electromagnetic spectrum as well as with the intensities of the radiation. This implies the separation of the spectral components into controlled intervals and power measurement for each interval using a spectrometer - a sophisticate and expensive piece of equipment.

The need of a simple method to obtain equivalent results for electrical lamp characterization lead to the method here described.

The digital camera is used for the light power measurements. For the spectral separation we use the classical reflective color chart approach: the incident light is filtered by the different color regions on the chart, in a fixed relationship with the incident light.

To use the setup, a full calibration of both the camera and the chart must be accomplished. A simple method is envisaged, in order to allow for easy chart recalibration either for its

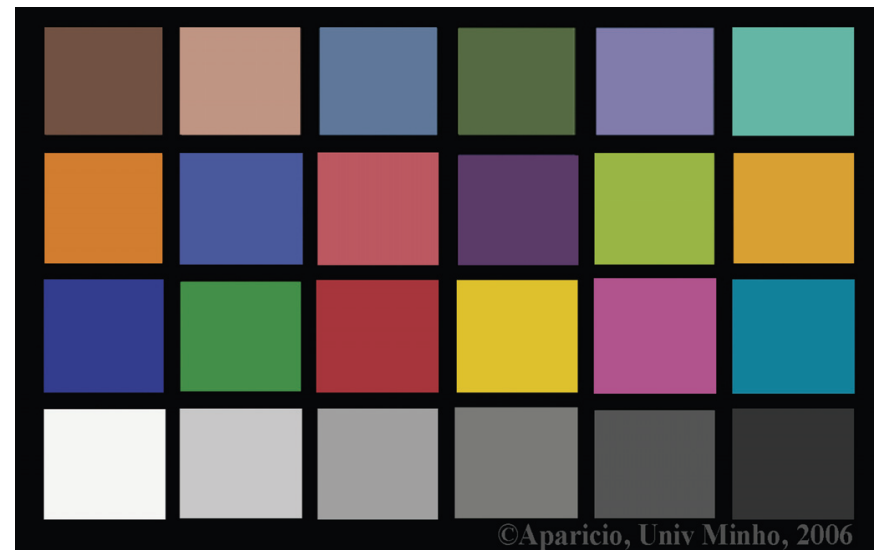

Fig. 1 - Reflective color chart reference

degradation in time or replacement for a new copy obtained from the digital image of the chart in another photo printing equipment, possibly not exactly matched to the prior one.

\section{THE REFERENCE CHART}

The reference chart uses reflection areas with different reflection coefficients. In the example in Fig. 1, we placed six grey squares from almost white to almost black in sequence at the bottom [2]. This is the main area, from where the calibration departs. The other color same-size squares are intended to cover the color spectrum as much as possible: next row comprises the basic colors components (red-green-blue and cyan-magenta-yellow) and the other two are a mix selection of colors. The borders are in black to avoid light to fool the camera exposure measurement.

This image was put in a jpeg file and taken to a commercial photo finishing machine produce several printed charts in various sizes.

\section{THE CAMERA}

The chart is photographed in the standard approach: steady mounted camera and chart, camera facing the chart, at a distance to practically cover the useful picture area with the chart image, lens optical axis orthogonal to the chart plane.

Any camera able to be focused to the chart and with manual settings for the light quality and exposure can be used. We had at hand a Nikon D1, a Coolpix 4200 and a Konica-Minolta A100. 
The illumination was by daylight - the standard natural reference - for the initial calibration.

Images with different exposures were taken, at 1/8, 1/4, 1/2, 1,2 and 4 times the correct exposure $(-3,-2,-1,0,+1,+2$ in relative exposure values $\mathrm{EV}$ ).

The images were saved in the usual jpeg file format at the best quality and transferred to a computer with the image analysis program.

\section{IMAGE ANALYSIS}

The images were filtered and average values of $5 \times 5$ areas were taken at the centers of the respective square spots in Lab mode (Photoshop). The results for the six grey areas are in Table 1.

The succession of the values in each row relates to the light intensity variation on the sensor due to the differences in exposures. Differences in the columns relate to different intensities due to different reflections for the spots in the same image.

TABLE I

LAB VALUES FOR THE GREY SPOTS

\begin{tabular}{|c|c|c|c|c|c|c|}
\hline \multirow{2}{*}{ Spot \# } & \multicolumn{6}{|c|}{ Exposure } \\
\cline { 2 - 7 } & -3 & -2 & -1 & 0 & +1 & +2 \\
\hline 1 & 4 & 7 & 16 & 28 & 43 & 59 \\
\hline 2 & 10 & 17 & 30 & 45 & 67 & 83 \\
\hline 3 & 17 & 27 & 43 & 63 & 89 & 98 \\
\hline 4 & 24 & 35 & 54 & 76 & 96 & 99 \\
\hline 5 & 30 & 43 & 65 & 89 & 99 & 100 \\
\hline 6 & 35 & 48 & 72 & 93 & 99 & 100 \\
\hline
\end{tabular}

The difference between consecutive exposures is known the light amount was doubled for each consecutive exposure the result can be plotted in a $\log \mathrm{x}$ scale, as in Fig. 2, were $1^{\text {st }}$ value in the $\mathrm{x}$-axis corresponds to exposure $-3 \mathrm{EV}$ (1/8 of the normal exposure), the 4 th to $0 \mathrm{EV}$ (normal exposure) and the $6^{\text {th }}$ and last to $+2 \mathrm{EV}$ ( $4 \mathrm{x}$ normal exposure).

\section{RESPONSE CHARACTERIZATION}

Early quantitative studies of the classical photographic process were presented by Hurter and Driffield in 1890 in a classic paper [4]. Their proposed method introduced the H\&D curve (also known as D-log E) relating optical density with the logarithm of the exposure. The typical photochemical sensitive material behavior produces an S-curve, with a linear central part, surrounded by the 'toe' and 'shoulder' regions. The slope of the function in the linear region is named 'gamma' and relates to the contrast of the process. Typical values are from 0.8 to 1.2 for negatives and 1.5 for transparencies.

With the advent of the electronic image systems for television, the main output device was the cathode ray tube (CRT). But it introduced one difficulty: it has a non-linear response $[1,2,5]$ empirically adjusted by a power-law as in equation 1 .

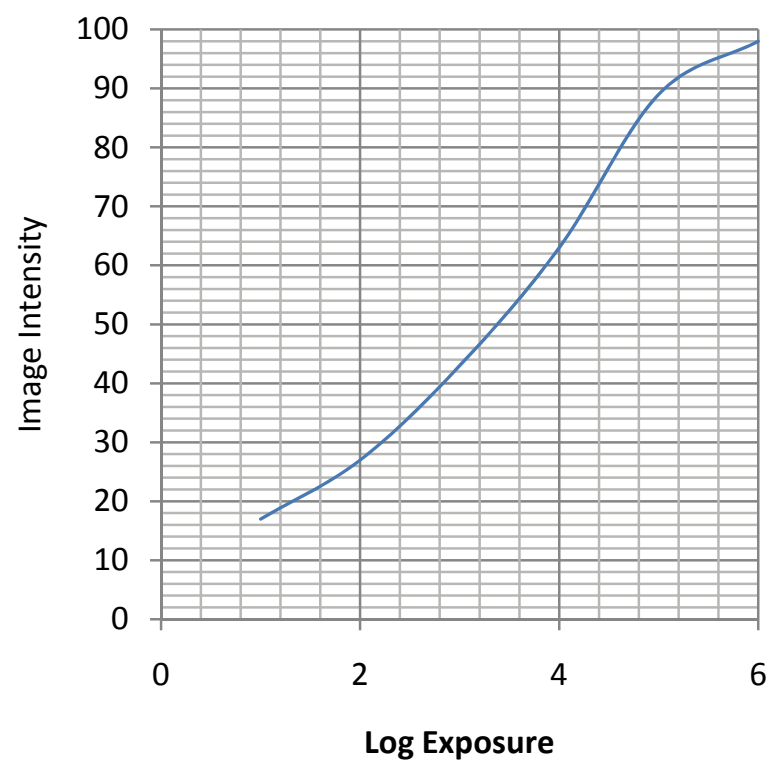

Fig. 2 - Graphic for spot \#3

$$
u=x^{\text {Gamma }}
$$

Practical values for Gamma are around 2.2. Coding the electric signal by a similar function using the inverse of the exponent, the net result is approximately linear.

The human sensor response is logarithmic and coding the output in a log-scale, the power function translates into a linear representation, and 'Gamma' is the slope - just like in the H\&D representation.

Changing the vertical scale of the graph in Fig. 2 from linear to logarithmic, the result is in Fig. 3.

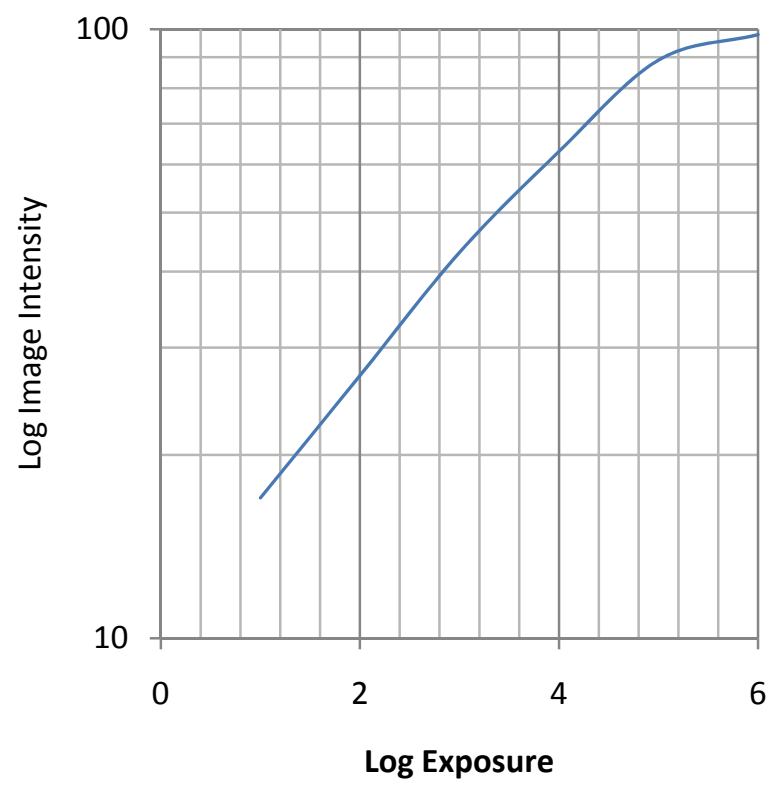

Fig. 3- Graphic for spot \#3 using vertical Log scale 
From the graphic, the relationship between the logarithms of the exposure $-\mathrm{x}$ axis - and image intensity $-\mathrm{y}$ axis - is mostly linear. It also presents a shoulder in the upper part (high intensities) just like in the classical photographic film approach. However, the low intensities do not present the equivalent 'toe' zone because there are no samples for very low values.

\section{SENSOR RESPONSE CALIBRATION}

Ploting the intensities variation acording to exposure for all the spots, there should be a horizontal displacement between the lines corresponding to the reflectance ratios between spots (Fig. 4). The corresponding exposure ratios should match the reflectance's.

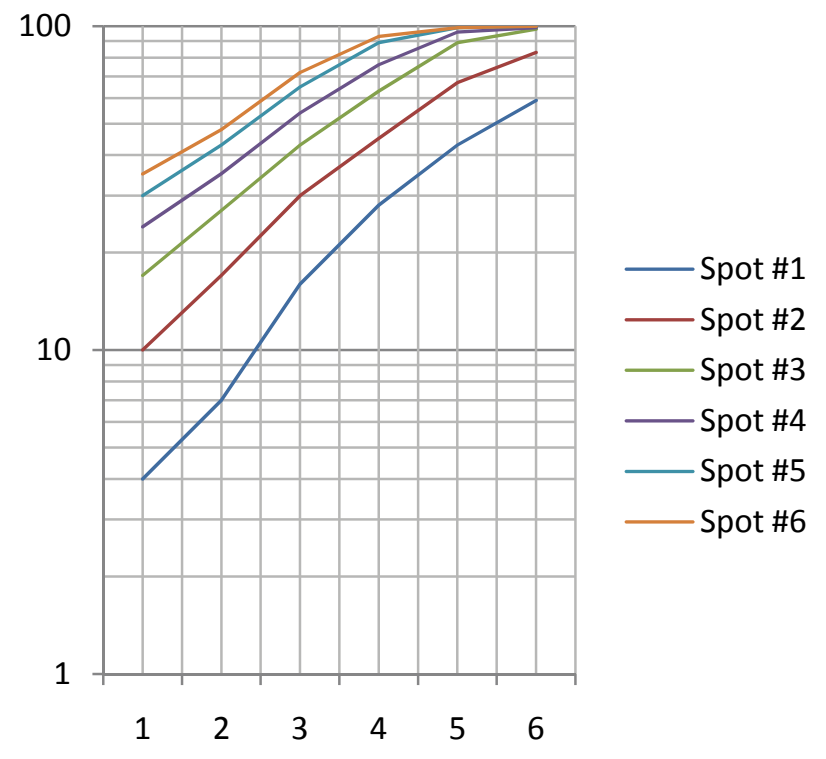

Fig. 4 - Graphic for all spots with vertical log scale

Plotted results show minor differences to the ideal situation. This can be due mainly because of image values reading errors, but also to camera exposure errors, noise problems or even camera processing differences related to the non-standard exposure choices.

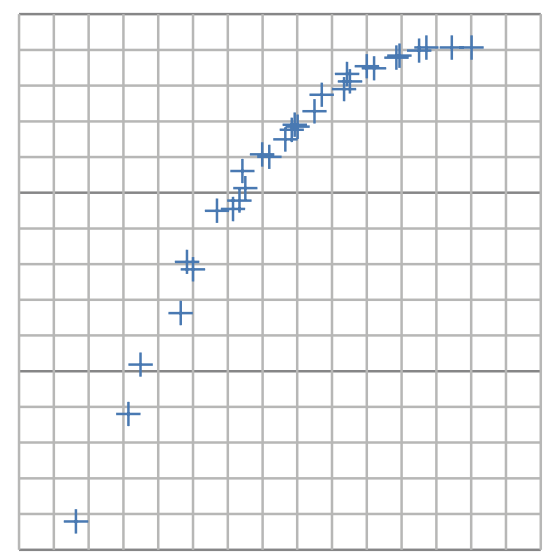

Fig. 5 - Log-log Camera/sensor response (relative values)
Probably, the cheapest cameras cannot provide the needed accuracy in exposure control or the manual adjustment ranges necessary for the method.

But, globally, an exposure shift between spots can be computed. The ratios were $1.3,1.4,1.5,1.8$ and 2.4 for each consecutive pair of spots.

Taking Spot \#6 (the lightest) as reference, the ratios become $100,77,55,37,20$ and $8.5 \%$.

The global response of the sensor/camera system is plotted in Fig. 5 for all the available data points, after taking in account the calculated reflectance ratios of the 6 grey areas.

The global response in Fig. 5, although taken from a single set of images, is already quite acceptable, with only minor deviations from the expected smooth line. It can be further optimized by averaging data from a larger set of images.

A problem yet to consider is the effect on the global response that some in-camera image processing approaches may bring. For instance there is a notice of recent cameras that include some sort of dark area illumination compensation: the dark areas are processed by the inbuilt algorithm in such a way as to brighten up, trying the equivalent of a flash illumination. If this is the case for the camera in use, this option should be disabled.

\section{FUTURE WORK}

This work is part of a larger project [3] aiming to study and characterize the new breed of lamps, including the low-power fluorescent compacts but also the led-type approaches that start to appear. One of the objectives of this larger project is the development of an automatic system able to analyze the images and output the coefficients for the color chart and also the spectral profile of the light emitted by the lamps.

The need for the chart calibration arises from the fact that image colors fade in time. But with a calibration method, such as the one proposed, even a partially faded chart can be used.

A single image of the chart taken in standard conditions forced daylight type illumination settings, but not auto light type adjustment - will allow the direct camera calibration using intensities of grey spots 1-6.

The corresponding light intensities ratios, computed from the RGB components relative to a standard color light condition such as daylight, translates into color components differences to identify the light source quality.

The colored spots correspond to different light source reflective filtering options and will allow further refinement of the process.

\section{ACKNOWLEDGMENT}

The research work is being conducted with partial funding from FCT (Portuguese Foundation for Science and Technology).

\section{REFERENCES}

[1] J. C. A. Fernandes, "Linear versus Non-Linear Digital Image Processing Methods," presented at Congresso Luso-Moçambicano de Engenharia, Maputo, Moçambique, 2005. 
[2] J. C. Aparício Fernandes , J. A. B. Campos Neves , Manuel J. S. Freitas J. L. Afonso, "Calibration of Camera for Light Quality Measurements of Low-Power Lamps ", 10th Portuguese-Spanish Conference In Electrical Engineering - XCLEEE, 5th - 7th of July 2007, Funchal, Madeira, Portugal

[3] Research proposal - PTDC/EEA-ENE/72551/2006 - Power Consumption and Spectral Radiance Characterization for Energy Saving Lamps, FCT, Portugal, 2006
[4] Hurter, Ferdinand and Driffield, Vero Charles, "Photochemical Investigations and a New Method of Determination of the Sensitiveness of Photographic Plates", J. Soc. Chem. Ind. May 31, 1890.

[5] Poynton, C., "The rehabilitation of gamma", in Rogowitz,B. E. and T. N. Pappas (eds.), Human Vision and Electronic Imaging III, Proceedings of SPIE 3299, 232-249 ,Bellingham, Wash.: SPIE, 1998. 